BULLETIN OF THE

AMERICAN MATHEMATICAL SOCIETY

Volume 77, Number 4, July 1971

\title{
A NONSTANDARD REPRESENTATION OF MEASURABLE SPACES AND $L_{\infty}$
}

\author{
BY PETER A. LOEB ${ }^{1}$
}

Communicated by Paul Cohen, November 9, 1970

The results given in this note were obtained by applying to measure theory the methods of nonstandard analysis developed by Abraham Robinson [5]. Amplifications of these results with proofs will be published elsewhere. ${ }^{2} \mathrm{It}$ is shown here that there are linear mappings from an arbitrary, real $L_{\infty}$ space and its dual $L_{\infty}^{*}$ into Euclidean $\omega$-space $E^{\omega}$, where $\omega$ is an infinite integer. Finite valued, finitely additive measures on the underlying measurable space are also mapped onto elements of $E^{\omega}$, and integrals are infinitesimally close to the corresponding inner products in $E^{\omega}$. Yosida and Hewitt's representation of $L_{\infty}^{*}[6]$ is an immediate consequence of these results.

In general, we use Robinson's notation [5]. If we have an enlargement of a structure that contains the set $R$ of real numbers, then ${ }^{*} R$ denotes the set of nonstandard real numbers and $* N$, the set of nonstandard natural numbers. A set $S$ is called ${ }^{*}$ finite if there is an internal bijection from an initial segment of $* N$ onto $S$; a *finite set has all of the "formal" properties of a finite set. Given $b$ and $c$ in ${ }^{*} R$, we write $b \simeq c$ if $b-c$ is in the monad of 0 ; when $b$ is finite, we write ${ }^{\circ} b$ for the unique, standard real number in the monad of $b$.

1. The partition $P$ and bounded measurable functions. Let $X$ be an infinite set and $\mathscr{T} C$ an infinite $\sigma$-algebra of subsets of $X$. Fix an enlargement of a structure that contains $X, \mathfrak{T}$, and the extended real numbers. There is a *finite, * ${ }^{*}$-measurable partition $P$ of $* X$ such that $P$ is finer than any finite $\mathbb{T}(-$ measurable partition of $X$. That is, $P C^{*} \mathscr{T} \mathrm{T}$ has the following properties:

(i) There is an infinite integer $\omega_{P} \in^{*} N$ and an internal bijection from $I=\left\{i \in{ }^{*} N: 1 \leqq i \leqq \omega_{P}\right\}$ onto $P$. Thus we may write $P=\left\{A_{i}: i \in I\right\}$.

(ii) If $i$ and $j$ are in $I$ and $i \neq j$, then $A_{i} \neq \varnothing$ and $A_{i} \cap A_{j}=\varnothing$.

AMS 1970 subject classifications. Primary 26A98, 28A60; Secondary 28A20, 28A25.

Key words and phrases. Measurable spaces, *finite partition, Euclidean $\omega$-space, finitely additive measures, purely finitely additive measures, representation of $L_{\infty}^{*}$, conditional expectation.

1 This work was supported by N.S.F. Grant NSF GP 14785.

2 These results were announced at the 1970 Oberwolfach conference on nonstandard analysis. 
(iii) $* X=\bigcup_{i \in I} A_{i}$.

(iv) For each $B \in \mathscr{M}$, let $I_{B}=\left\{i \in I: A_{i} \subset^{*} B\right\}$. Then $I_{B}$ is *finite, and ${ }^{*} B=\bigcup_{i \in I_{B}} A_{i}$.

(v) Let $M$ be the set of $\mathfrak{T}$-measurable functions on $X$, and $M B$, the set of bounded functions in $M$. For each $f \in M B$ and $i \in I$, $\sup _{x \in A_{i}} * f(x)-\inf _{x \in A_{i}} * f(x) \simeq 0$.

Given the partition $P$, we let $E$ denote the set of all internal mappings from $I$ into $* R$. The set $E$ has all of the "formal" properties of Euclidean $n$-space. We shall write $x_{i}$ instead of $x(i)$ for $x \in E$ and $i \in I$, and we shall write $x \cong y$ if $x, y \in E$ and $x_{i} \simeq y_{i}, \forall i \in I$. Let $c_{P}$ denote a fixed internal choice function defined on $I$ with $c_{P}(i) \in A_{i}$ $\in P$ for each $i \in I$. Let $T$ denote the mapping from $M B$ into $E$ defined by setting $T(f)(i)={ }^{*} f\left(c_{P}(i)\right)$ for each $f \in M B$ and $i \in I$.

Proposition 1. Given $f, g$ in $M B$ and $\alpha, \beta$ in $R, T(\alpha f+\beta g)=\alpha T(f)$ $+\beta T(g)$ and $T(f) \neq T(g)$ if $f \neq g$.

2. Measures and integration. Let $\Phi(X, \mathfrak{T})$, or simply $\Phi$, denote the set of all finitely additive real-valued functions $\mu$ on $\mathfrak{T}$ such that $\sup _{B \in \mathfrak{M}}|\mu(B)|<+\infty$. Let $U$ be the mapping of $\Phi$ into $E$ defined by setting $U(\mu)(i)={ }^{*} \mu\left(A_{i}\right)$ for each $\mu \in \Phi$ and $i \in I$. Clearly, $U$ preserves addition and multiplication by real numbers. Conversely, if $e \in E$ and both $\sum_{i \in I}\left(e_{i} \bigvee 0\right)$ and $\sum_{i \in I}\left(-e_{i} \bigvee 0\right)$ are finite in $* R$, let $\varphi(e)$ be that element of $\Phi$ such that for each $B \in \mathscr{N}, \varphi(e)(B)={ }^{\circ} \sum_{i \in I_{B}} e_{i}$. (Note that we are writing $\sum$ instead of $* \sum$ for the extension of the summation operator.) For each $\mu \in \Phi, \varphi(U(\mu))=\mu$, but in general, $U(\varphi(e)) \approx e$. If $\mu$ and $\nu$ are in $\Phi$, then $U(\mu) \wedge U(\nu) \cong U(\mu \wedge \nu)$, and - $\sum_{i \in I}|U(\mu)(i)|=|\mu|(X)$.

Let $\Phi_{c}$ and $\Phi_{p}$ be, respectively, the set of countably additive and the set of purely finitely additive elements of $\Phi$. Yosida and Hewitt's Theorem 1.19 [6] has the following extension:

THEOREM 1. There is a set $K \in^{*} \mathfrak{T}$ such that for all $\mu \in \Phi_{c},\left|{ }^{*} \mu\right|(K)$ $\simeq 0$ and for all $\nu \in \Phi_{p},\left|{ }^{*}\right|\left({ }^{*} X-K\right)=0$.

Without loss of generality, we assume that $K=\bigcup\left\{A_{i} \in P: A_{i} \subset K\right\}$. If $\mu=\mu_{c}+\mu_{p}$ is the decomposition of an element $\mu$ in $\Phi=\Phi_{c} \oplus \Phi_{p}$, then when $A_{i} \subset^{*} X-K, \quad U(\mu)(i)=U\left(\mu_{c}\right)(i)$ and when $A_{i} \subset K, \quad U(\mu)(i)$ $\simeq U\left(\mu_{p}\right)(i)$. We next show that there is a "maximum" null set for each $\mu \in \Phi^{+}$, and we extend the Hahn decomposition theorem for countably additive signed measures.

THEOREM 2. Let $\mu$ be an arbitrary, finitely additive signed measure on $(X, \mathfrak{T l})$. Let 


$$
A_{+}=\bigcup\left\{A_{i} \in P:{ }^{*} \mu\left(A_{i}\right)>0\right\}, A_{-}=\bigcup\left\{A_{i} \in P:{ }^{*} \mu\left(A_{i}\right)<0\right\},
$$

and

$$
A_{0}=\bigcup\left\{A_{i} \in P:{ }^{*} \mu\left(A_{i}\right)=0\right\} .
$$

Then ${ }^{*} \mu\left(A_{0}\right)=0$, and for each $\mu$-null set $B \in \mathfrak{T l},{ }^{*} B \subset A_{0}$. If there exists a $\mu$-positive set $B_{+}$and a $\mu$-negative set $B_{-}$in $\mathfrak{T l}$ with $X=B_{+} \cup B_{-}$and $B_{+} \cap B_{-}=\varnothing$, then $A_{+} \subset^{*} B_{+}, A_{-} \subset^{*} B_{-}$, and each $A_{i} \in P$ is either a ${ }^{*} \mu$-positive set or a ${ }^{*} \mu$-negative set.

If we apply Theorem 2 to Lebesgue measure on the real line, we see that every standard real number is in the null set $A_{0}$.

Let $\Phi_{1}=\{\mu \in \Phi: \mu(X)=1$ and $\forall B \in \Re, \mu(B)=0$ or $\mu(B)=1\}$. For each $j \in I$, let $\delta^{j} \in E$ be defined by setting $\delta_{i}^{j}=0$ if $i \neq j$ and $\delta_{j}^{j}=1$.

THEOREM 3. For each $j \in I, \varphi\left(\delta^{j}\right) \in \Phi_{1}$, and for each $\mu \in \Phi_{1}, U(\mu)=\delta^{j}$ for some $j \in I$. Moreover, if $\{x\} \in \mathbb{N}$ for each standard point $x \in X$, then the following are equivalent statements:

(i) Given $j \in I, \varphi\left(\delta^{j}\right) \in \Phi_{p}$ iff $A_{j} \neq\{x\}$ for any standard point $x \in X$.

(ii) Every free $\mathfrak{T}$-measurable ultrafilter $\mathscr{F} \subset \mathfrak{N}$ contains a chain $B_{1} \supset B_{2} \supset \cdots$, with $\bigcap_{n=1}^{\infty} B_{n}=\varnothing$.

If $\mu$ is a nonnegative finitely additive measure on $(X, \mathfrak{T})$ and $f \geqq 0$ is $\mu$-integrable on $X$, then for each $B \in \mathscr{N}$,

$$
\int_{B} f d \mu \simeq \sum_{i \in I_{B}}\left(\inf _{x \in A_{i}} *_{f}(x)\right) *_{\mu}\left(A_{i}\right) .
$$

We can relate integration on $X$ to the inner product "." in $E$ as follows:

Theorem 4. If $f \in M B$ and $\mu \in \Phi$, then for each $B \in \mathfrak{M}$,

$$
\int_{B} f d \mu={ }_{i \in I_{B}}{ }^{*} f\left(c_{P}(i)\right) *\left(A_{i}\right) .
$$

In particular, $\int_{X} f d \mu \simeq T(f) \cdot U(\mu)$.

In general, Theorem 4 is false for unbounded functions $f \in M$. One can, however, find for each $f \in M$ an $\omega \in{ }^{*} N$ such that if ${ }^{*} f_{\omega}=-\omega \bigvee^{*} f$ $\wedge \omega$, then for each $i \in I, \sup _{x \in A_{i}} *^{*} f_{\omega}(x)-\inf _{x \in A_{i}}{ }^{*} f_{\omega}(x) \simeq 0$. If $\mu \in \Phi$ and $f$ is $\mu$-integrable, then

$$
\int_{X} f d \mu \simeq \sum_{i \in E} f_{\omega}\left(c_{P}(i)\right)^{*} \mu\left(A_{i}\right) .
$$

3. The space $L_{\infty}$ and its conjugate space. Let $\mathscr{T}$ be a proper subfamily of $\mathscr{T}$ such that $\mathscr{N}$ is closed under the formation of countable 
unions and every $\mathscr{T C}$-measurable subset of an element of $\mathscr{T}$ is an element of $\Re$. For each $f \in M$, set

$$
\|f\|_{\infty}=\inf \{\alpha \in R:\{x \in X:|f(x)|>\alpha\} \in \mathscr{N}\},
$$

and let $M_{0}=\left\{f \in M:\|f\|_{\infty}<+\infty\right\}$. We say that two functions $f$ and $g$ in $M_{0}$ are equivalent if $\|f-g\|_{\infty}=0$, and we let $L_{\infty}$ denote the usual Banach space of equivalence classes in $M_{0}$ with norm $\|\cdot\|_{\infty}$.

Given $\mathscr{N}$, let $I_{0}=\left\{i \in I: A_{i} \in \in^{*}\right\}$. Clearly, if $B \in \mathscr{N}, I_{B} \subset I_{0}$. For each $f \in M_{0}$, let $T_{0}(f)$ be that element of $E$ such that $T_{0}(f)(i)={ }^{*} f\left(c_{P}(i)\right)$ for $i \in I-I_{0}$ and $T_{0}(f)(i)=0$ for $i \in I_{0}$. Given $f$ and $g$ in $M_{0}$, $T_{0}(f) \cong T_{0}(g) \Rightarrow\|f-g\|_{\infty}=0 \Rightarrow T_{0}(f)=T_{0}(g)$. Moreover, $\|f\|_{\infty} \simeq$ $\max _{i \in I}\left|T_{0}(f)(i)\right|$. We may, therefore, consider $T_{0}$ to be a mapping of $L_{\infty}$ into $E$; this mapping preserves addition and multiplication by standard real numbers.

For each functional $F$ in the dual space $L_{\infty}^{*}$ of $L_{\infty}$, let $V(F)$ be the element of $E$ such that for all $i \in I, V(F)(i)={ }^{*} F\left(\chi_{A_{i}}\right)$, and let $\mu_{F}$ $=\phi(V(F))$. It is easy to see that $U\left(\mu_{F}\right)=V(F)$. Yosida and Hewitt's representation of $L_{\infty}^{*}([6$, p. 53]) now has the following form:

Theorem 5. Let $\Phi_{0}$ be the normed vector space $\{\mu \in \Phi: \mu(B)=0$, $\forall B \in \mathfrak{N}\}$ with norm given by $\|\mu\|=|\mu|(X)$. For each $F \in L_{\infty}^{*}$, let $\Theta(F)=\mu_{F}$. Then $\Theta$ is an isometric isomorphism from the Banach space $L_{\infty}^{*}$ onto $\Phi_{0}$, and for each $F \in L_{\infty}^{*}$ and $f \in L_{\infty}$ we have

$$
F(f)=\int_{X} f d \mu_{F} \simeq V(F) \cdot T_{0}(f) .
$$

Corollary. $A$ nonzero functional $F \in L_{\infty}^{*}$ is multiplicative iff $U\left(\mu_{F}\right)=\delta^{j}$ for some $j \in I-I_{0}$.

Assume now that there is a nonnegative $\mu \in \Phi_{c}$ such that $\mathscr{x}$ $=\{B \in \mathfrak{T}: \mu(B)=0\}$. If $f \in L_{\infty}$ and $\nu \in \Phi_{c}$ has the value $\nu(B)=\int_{B} f d \mu$ for each $B \in \mathbb{T}$, then for each $i \in I-I_{0},{ }^{*} f\left(c_{P}(i)\right) \simeq{ }^{*} \nu\left(A_{1}\right) /{ }^{*} \mu\left(A_{i}\right)$. To apply this result to probability theory, assume that $\mu(X)=1$ and choose a $\sigma$-algebra $\mathfrak{M}_{1} \subset \mathfrak{M}$. There is a ${ }^{*}$ finite, ${ }^{*} \mathfrak{M}_{1}$-measurable partition $P_{1}$ of $* X$ such that $P_{1}$ is finer than any standard, finite $\Re_{1}$ measurable partition of $X$ and such that for each $C \in P_{1}, C$ $=\bigcup\left\{A_{i} \in P: A_{i} \subset C\right\}$. If $Y \in M B$ and $E\left(Y, \mathfrak{T}_{1}\right)$ is the conditional expectation of $Y$ with respect to $\Re_{1}$, then for each $C \in P_{1}$ with $\mu(C) \neq 0$ and for each $x \in C$,

$$
{ }^{*} E\left(Y, \mathfrak{T}_{1}\right)(x) \simeq\left[\sum_{A_{i} \in P ; A i \subset C} * Y\left(c_{P}(i)\right){ }^{*} \mu\left(A_{i}\right)\right] /{ }^{*} \mu(C) .
$$




\section{REFERENCES}

1. E. Berkson and H. Porta, Representations of $\mathfrak{B}(X)$, J. Functional Analysis 3 (1969), 1-34. MR 38 \#3728.

2. N. Dunford and J. T. Schwartz, Linear operators. I: General theory, Pure and Appl. Math., vol. 7, Interscience, New York, 1958. MR $22 \# 8302$.

3. W. A. J. Luxemburg (Editor), Applications of model theory to algebra, analysis, and probability, Holt, Rinehart and Winston, New York, 1969. MR 38 \#3143.

4. M. Machover and J. Hirschfeld, Lectures on nonstandard analysis, Lecture Notes in Math., no. 94, Springer-Verlag, Berlin and New York, 1969. MR 40 \#2531.

5. A. Robinson, Non-standard analysis, North-Holland, Amsterdam, 1966. MR $34 \# 5680$.

6. K. Yosida and E. Hewitt, Finitely additive measures, Trans. Amer. Math. Soc. 72 (1952), 46-66. MR 13, 543.

UNIVERSITY OF ILLINOIS, URBANA, ILLINOIS 61801 